\title{
Early experience with Millipede IRIS transcatheter mitral annuloplasty
}

\author{
Jason H. Rogers ${ }^{1}$, Walter D. Boyd ${ }^{2}$, Thomas W. Smith ${ }^{1}$, Steven F. Bolling ${ }^{3}$ \\ ${ }^{1}$ Division of Cardiovascular Medicine, ${ }^{2}$ Division of Cardiothoracic Surgery, University of California, Davis Medical Center, Sacramento, CA, USA; \\ ${ }^{3}$ Department of Cardiac Surgery, University of Michigan Health System, Ann Arbor, MI, USA \\ Correspondence to: Jason H. Rogers, MD. Division of Cardiovascular Medicine, University of California, Davis Medical Center, Sacramento, CA, \\ USA. Email: jhrogers@ucdavis.edu.
}

\begin{abstract}
The IRIS mitral annuloplasty ring is a transcatheter, transfemoral and transseptal-delivered complete, semirigid annuloplasty ring. The IRIS system mimics surgical annuloplasty by reducing the mitral septal-lateral dimension and improving leaflet coaptation. We report the early experience with the IRIS system in seven patients. These patients had 3-4+ mitral regurgitation (MR) with annular dilation and were symptomatic NYHA II-IV with LV end systolic dimensions $\leq 65 \mathrm{~mm}$. Patients were excluded for LVEF $<20 \%$, aortic valve disease, right-sided heart failure and PA systolic pressure $>70 \mathrm{mmHg}$. Baseline and 30-day transthoracic echocardiography and CT imaging was performed. In phase 1, 4 patients had surgical IRIS mitral ring implantation. In phase 2, 3 patients had transfemoral, transseptal delivery of the IRIS mitral ring. There was no procedural death, or MI. The mitral SL diameter was reduced from $38.0 \pm 4.1$ to $25.9 \pm 4.9 \mathrm{~mm}$ at 30 days (31.8\% SL reduction, $n=7)$. MR was reduced from baseline $3-4+$ to $0-1+$ in all patients at 30 days. There were improvements in NYHA class and there was a decrease in diastolic LV volumes from $182.4 \pm 54.3$ to $115.3 \pm 98.8 \mathrm{~mL}$ at 30 days (36.8\% reduction). Based on these initial positive findings, ongoing clinical trials are underway to further evaluate the safety and efficacy of the IRIS ring.
\end{abstract}

Keywords: Mitral regurgitation; transcatheter mitral valve repair; mitral annuloplasty

Submitted Oct 02, 2018. Accepted for publication Oct 09, 2018.

doi: $10.21037 /$ acs.2018.10.05

View this article at: http://dx.doi.org/10.21037/acs.2018.10.05

\section{Introduction}

Mitral valve annuloplasty was first described almost 50 years ago as a novel technique for mitral valve reconstruction (1). Since that time, use of annuloplasty has become the gold standard as part of successful surgical mitral valve (MV) repair. In patients with chronic mitral regurgitation (MR), structural heart changes including left atrial (LA) and left ventricular (LV) enlargement are common, leading to mitral annular dilation. This enlargement of the mitral annulus not only causes but contributes to MR progression over time. In primary MR, leaflet abnormalities (such as MV prolapse) are responsible for valve dysfunction.

Surgical repair consists of leaflet repair (plication or resection) and/or chordal replacement as needed. The addition of an annuloplasty ring is performed routinely and aids in the immediate effectiveness and durability of the repair. In secondary MR, the major abnormality is left ventricular dysfunction, which causes mitral annular enlargement and apical tethering of the MV leaflets, impairing coaptation in systole. In properly selected patients without excessive left ventricular size or leaflet tethering, mitral ring annuloplasty can be performed as stand-alone corrective therapy for secondary MR. A major goal for transcatheter mitral valve correction is to develop a "toolbox" that may one day reproduce the effective and durable repairs that can be achieved with surgery. Since many patients have significant co-morbidities and are not routinely offered surgery due to high surgical risk, developing transcatheter tools can address an unmet 
clinical need (2). The Millipede IRIS semi-rigid complete annular reshaping ring is the first complete transcatheter transfemoral annuloplasty ring to be used successfully for the treatment of MR.

\section{The Millipede IRIS device}

The Millipede IRIS annuloplasty ring (Millipede, Inc., Santa Rosa, CA, USA) is a semi-rigid, complete (i.e., closed) ring that is made from laser-cut and heat-set nickel-titanium alloy (nitinol). The ring has 8 helical stainless-steel anchors pre-attached to the base of the device. Each anchor rotates independently and attaches directly to the mitral annulus. Each anchor can also be retracted or "unscrewed" if the initial position is undesirable and maneuvered to a different

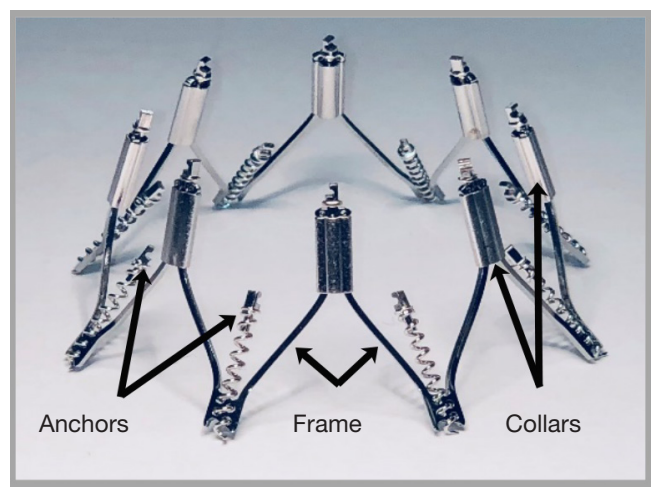

Figure 1 The transcatheter Millipede IRIS device. The Millipede IRIS device consists of a nitinol frame, 8 helical anchors that attach to the mitral annulus, and 8 sliding collars that allow actuation of the device and reduction of the mitral annular diameter. location. The upper portion of the device has 8 sliding collars which can be individually advanced or retracted. When tensioned, each collar brings the two adjacent helical anchors closer together (Figure 1). Since each collar shortens the associated segment of the ring, customized reduction in annular circumference and diameter can be achieved. By selectively tightening crowns individually, targeted annuloplasty can be performed to reduce the most dilated portions of the annulus. The 3 basic steps of the IRIS procedure are (I) placement, (II) anchoring and (III) actuation (Figure 2). Features of the device include the ability to completely reposition and retrieve the device until the decision is made to fully release the IRIS ring from the delivery system.

\section{Millipede IRIS implantation procedure}

Details of the first-in-human experience with the Millipede IRIS device have been previously published (3). In this report, patients treated with the IRIS device were screened and enrolled if the following inclusion criteria were met: (I) 3 or 4+ ischemic or non-ischemic, predominantly secondary MR with a dilated mitral annulus; (II) symptomatic NYHA class II, III or ambulatory IV; (III) LV end-systolic diameter $\leq 65 \mathrm{~mm}$; (IV) patients were suitable candidates surgical mitral valve correction. Exclusion criteria for this initial series were: (I) LV ejection fraction $<20 \%$, (II) untreated significant CAD or any revascularization within 30 days; (III) aortic valve disease requiring intervention; (IV) pulmonary artery systolic pressure $>70 \mathrm{mmHg}$; (V) right-sided congestive heart failure; (VI) any prior mitral or tricuspid valve surgery; (VII) active infection or life expectancy

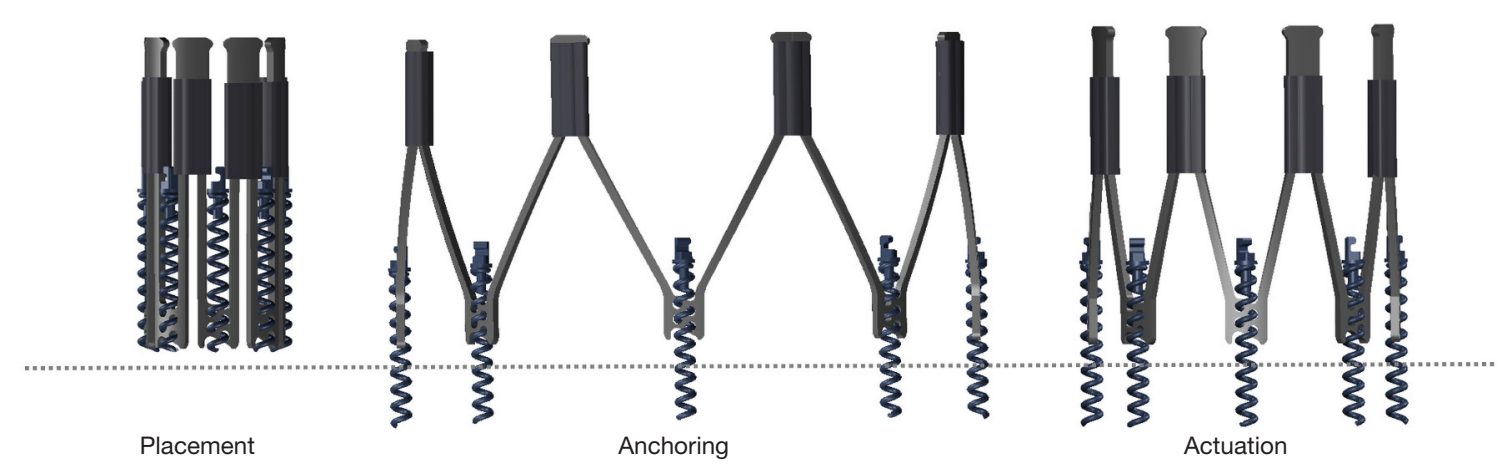

Figure 2 The transcatheter Millipede IRIS procedure. First, the IRIS ring is placed onto the mitral annulus. Next, the ring is opened and attached to the mitral annulus by sequentially rotating 8 helical anchors. Finally, the device is actuated by rotating the crown collars, which reduces the mitral annulus to the desired final diameter. 

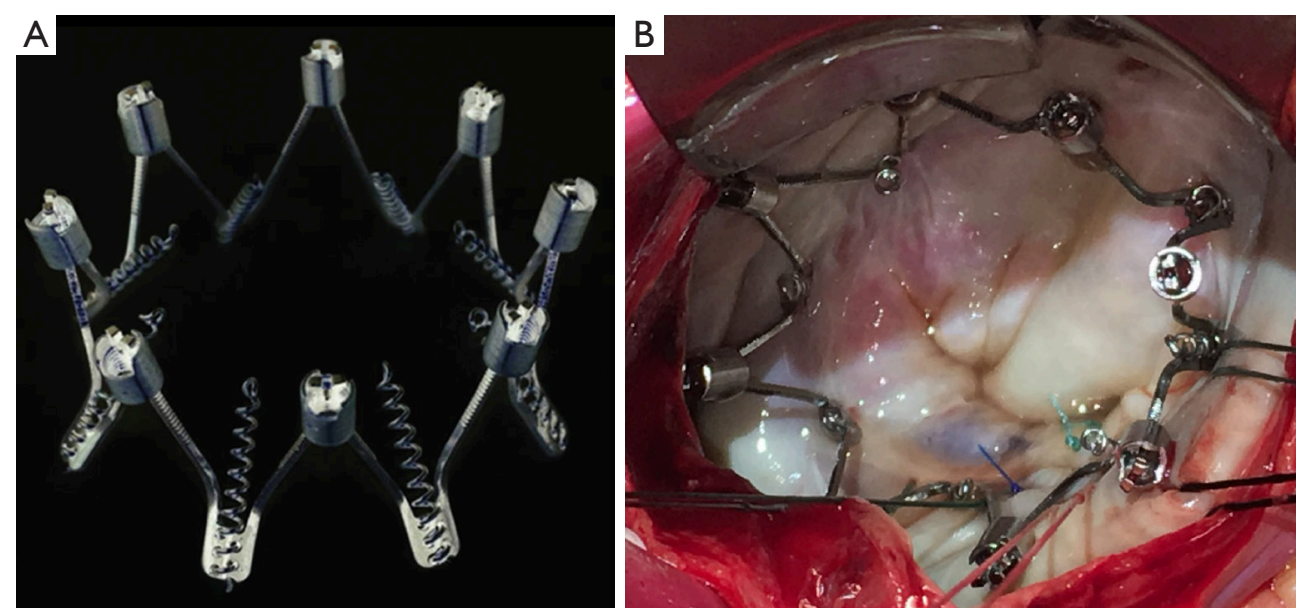

Figure 3 Surgical IRIS ring. The surgical IRIS ring (A), and operative appearance after placement on the mitral annulus (B). Reproduced with permission (3).

$<12$ months. Mitral annular dimensions were measured by transthoracic and transesophageal echocardiography and MR quantification was performed using the Mitral Valve Academic Research Consortium (MVARC) criteria (4). Gated cardiac computed tomography scans were performed for analysis of the left atrial (LA) and left ventricular (LV) diastolic volumes pre- and post-procedure (Clinical Imaging Analytics, Guerneville, CA, USA). Follow-up was performed at 30 days, with 6-month and 1-year planned follow-up.

\section{Surgical Millipede IRIS implants}

The first human implants of the Millipede IRIS were performed with a surgical device using median sternotomy, standard bicaval cannulation and cardiopulmonary bypass. The surgical implants (referred to as phase 1) were performed to establish the safety and efficacy of the implant prior to transcatheter delivery. The device was placed onto the mitral annulus using a short catheter and each helical anchor was rotated into the mitral annulus under direct vision (Figure 3). The collars were then rotated to actuate the device and reduce the mitral diameter until no MR was present by surgical "bulb irrigation" testing with saline. Three temporary implants prior to planned surgical annuloplasty were performed. This was followed by 4 patients treated with permanent implants in both the mitral $(n=4)$ and mitral + tricuspid $(n=2)$ position.

The next phase of the Millipede IRIS first-in-human series (phase 2) consisted of transfemoral, transseptal transcatheter delivery of a lower-profile transcatheter IRIS ring to the mitral annulus (Figure 4). These procedures were performed under general anesthesia in the cardiac catheterization laboratory. Right femoral vein access was obtained and pre-closed with two 6 French Proglide devices (Abbott, Santa Clara, CA, USA). Transseptal puncture was performed in the superior and posterior portion of the interatrial septum using standard techniques at a height of $\sim 4 \mathrm{~cm}$ above the plane of the mitral annulus. Over a $0.035 "$ wire, the IRIS deflectable guide catheter was advanced from the right femoral vein, across the septum and into the left atrium. The guide was aspirated and flushed and heparin was given to maintain an of ACT 250-300 seconds.

Next, the IRIS device, which was preloaded onto the 24 French deflectable IRIS delivery catheter, was advanced through the guide catheter. The IRIS device was then steered under fluoroscopic and echocardiographic guidance to a position superior to the annulus and centered over the mitral orifice. The device was expanded and anchored on the mitral annulus using both TEE and intracardiac echocardiography (ICE). ICE allows direct visualization of each individual anchor attachment in an optimal location on the mitral annulus. The IRIS device was then actuated to achieve the desired reduction in the mitral annular diameter. After confirming circumflex coronary patency by angiography, the IRIS device was released from the delivery catheter. The delivery catheter was then removed, followed by removal of the guide catheter. In the first 3 transcatheter 

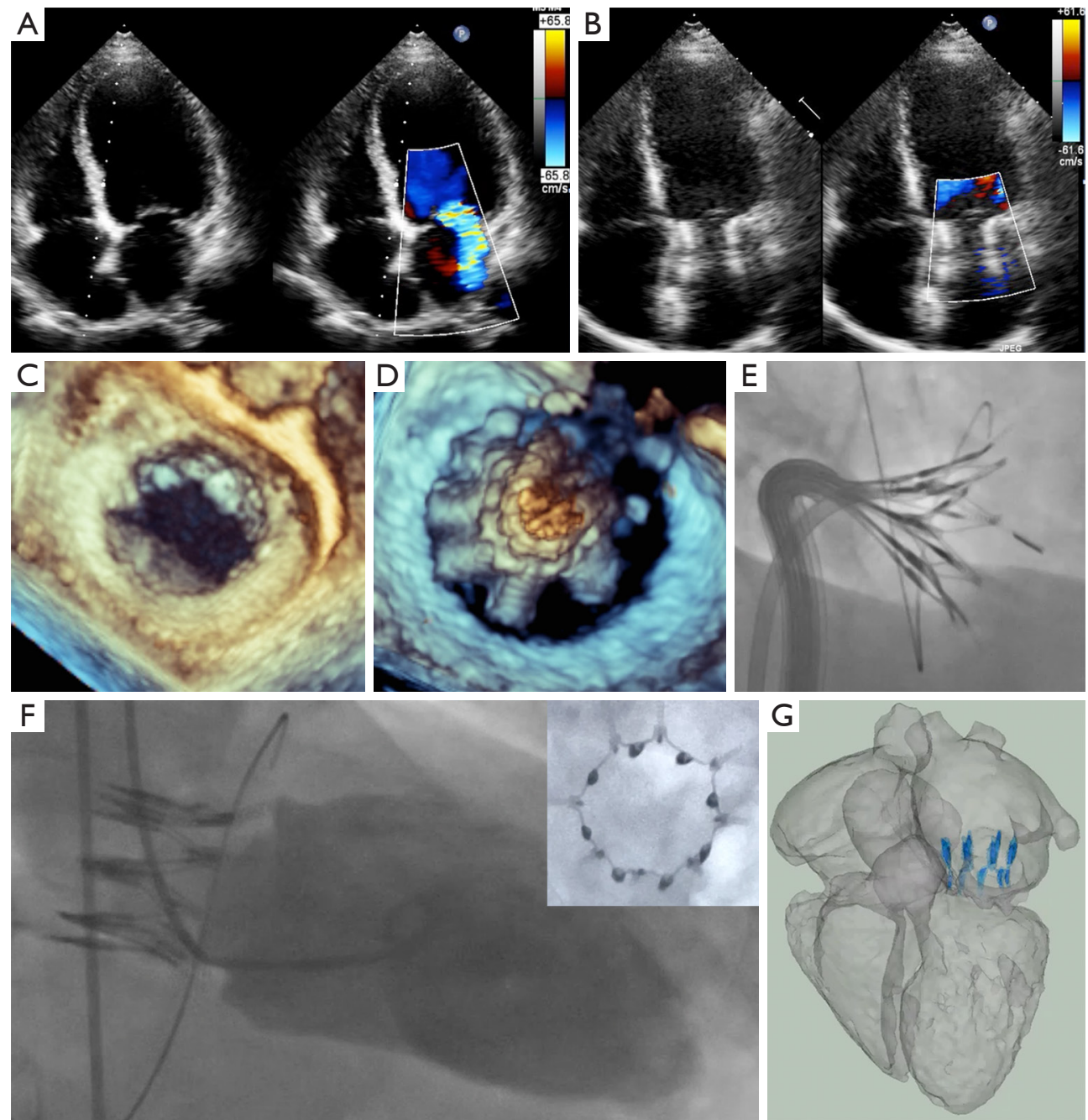

Figure 4 Transcatheter IRIS ring. (A) Preprocedure transthoracic echocardiogram showing 3+ secondary MR; (B) 30-day post-IRIS implantation with no MR; (C) baseline native mitral valve annulus on TEE; (D) centering the IRIS ring on the mitral valve prior to full expansion and anchoring; (E) fluoroscopic appearance of transfemoral transseptal delivery of IRIS ring; (F) final fluoroscopic appearance during left ventriculogram and en face view (inset) ; (G) CT scan at 30 days showing position of IRIS ring (blue) in the heart. Reproduced with permission (3).

cases, we elected to close all iatrogenic ASDs after the procedure with $10 \mathrm{~mm}$ Amplatzer septal occluders (Abbott, Santa Clara, CA) to eliminate any potential confounding influence from a residual ASD. The femoral vein was closed with the previously deployed Proglides.

\section{Millipede IRIS clinical results}

In the initial clinical report, 7 patients were included in phase $1(n=4)$ and phase $2(n=3)$ implants and all received the IRIS ring. The average age of the patients treated was 60.3 years, appropriate medical therapy was administered per the local heart team and all patients were candidates for surgical mitral valve annuloplasty. The first 4 patients in Phase 1 received the IRIS ring through a conventional open surgical approach. The subsequent 3 patients in Phase 2 received the transcatheter mitral IRIS rings to the mitral annulus and their average LVEF was $42 \% \pm 19 \%$. There was no device-related procedural death, stroke, or MI. In all of the patients who received the IRIS device, the pre- 

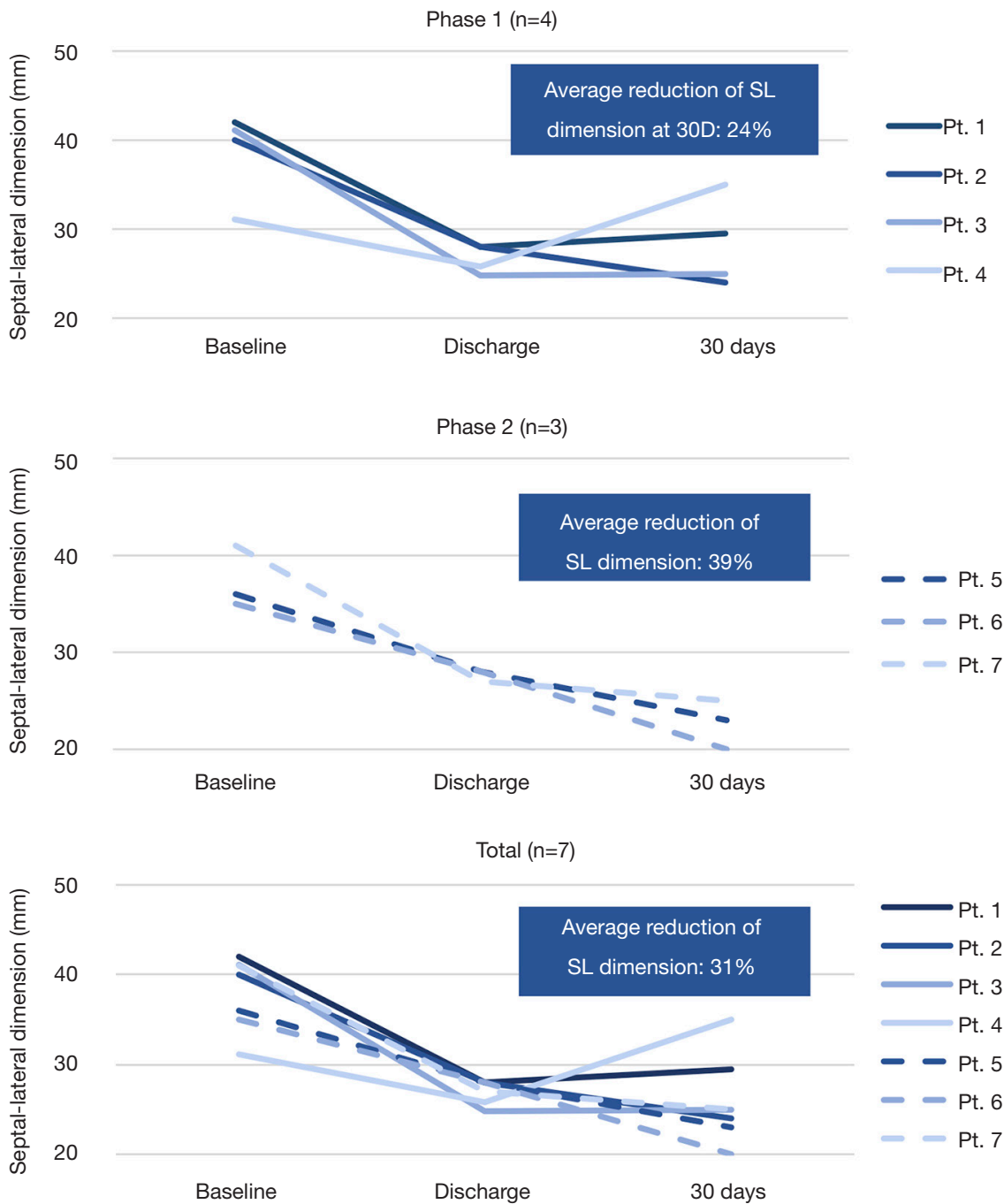

Figure 5 Reduction in septal-lateral dimension. Phase 1, surgical implants; Phase 2, transcatheter implants. Reproduced with permission (3).

procedure mitral SL diameter as determined by TTE was reduced from a baseline of $38.0 \pm 4.1$ to $25.9 \pm 4.9 \mathrm{~mm}$ at 30 days, representing an average SL reduction of $31.8 \%$ (Figure 5).

Every patient in the study demonstrated reduction of MR, with all patients showing a decline from a baseline of 3 or $4+M R$ to 0 or $1+M R$ at 30 days (Figure 6). There were improvements in NYHA class (Figure 7) and reductions in left ventricular diastolic volumes (Figure 8). There was a decrease in diastolic $\mathrm{LV}$ volumes from $182.4 \pm 54.3 \mathrm{~mL}$, to $115.3 \pm 98.8 \mathrm{~mL}$ at 30 days $(36.8 \%$ reduction) $(\mathrm{n}=7)$. The LA volume was $172.7 \pm 67.2 \mathrm{~mL}$ at baseline and $133.6 \pm 62.3 \mathrm{~mL}$ at 30 days $(\mathrm{n}=7)$.

\section{Discussion}

The Millipede IRIS ring is the first complete semi-rigid annuloplasty ring to be delivered by a percutaneous, transfemoral and transseptal route. The mechanism of action involves complete and circumferential annuloplasty and there were reductions in the mitral SL diameter, MR, $\mathrm{LV}$ volumes, and improvement in NYHA Class.

Mitral annuloplasty is used frequently during surgical mitral valve correction and is considered a current "gold standard" element of successful repair. Annuloplasty is routinely applied in two patient subgroups. First, restrictive annuloplasty, which utilizes an undersized ring to normalize 


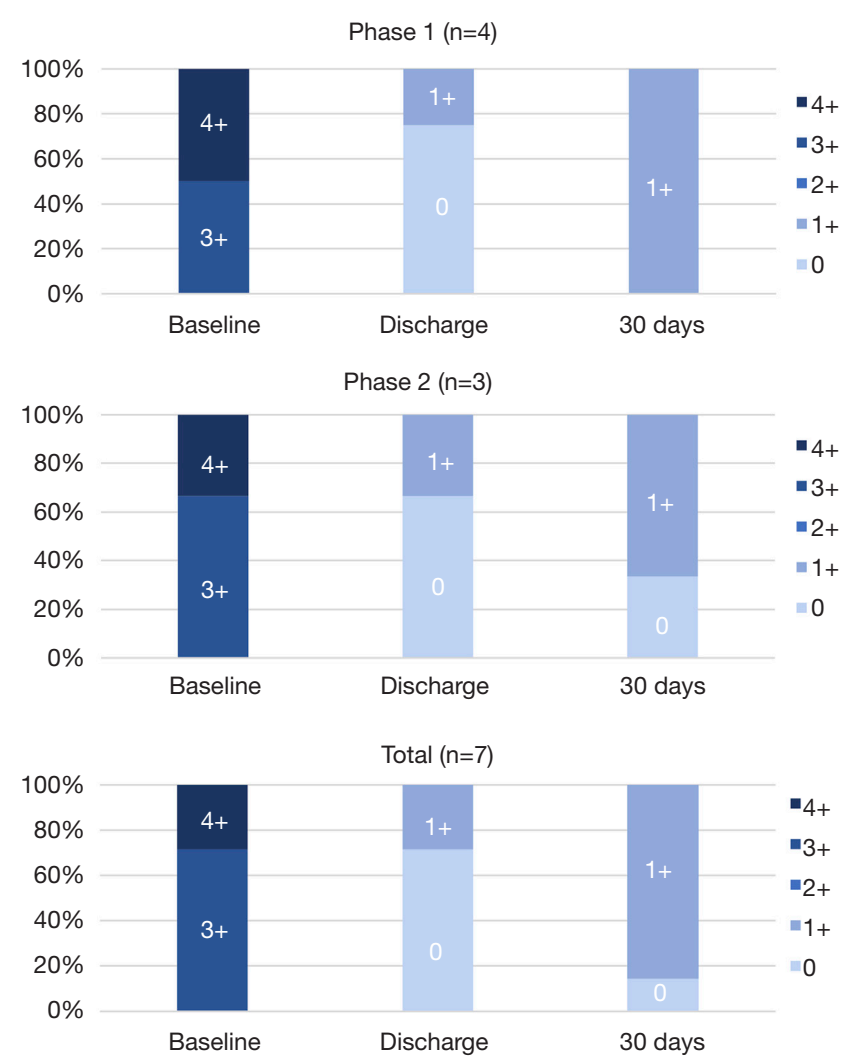

Figure 6 Mitral regurgitation grade. Reproduced with permission (3). Phase 1, surgical implants; Phase 2, transcatheter implants.

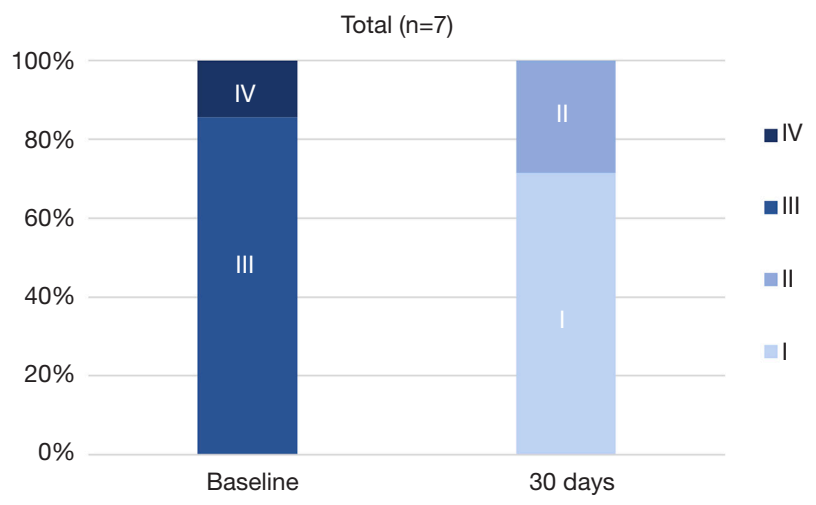

Figure 7 New York Heart Association (NYHA) Functional Class. Reproduced with permission (3).

the anterior-posterior mitral annular diameter, can be used as stand-alone therapy for secondary MR. Second, annuloplasty rings have been used in primary MR repair to stabilize the annulus, which has been shown to significantly improve surgical repair durability (5).

Prior studies have shown that the use of complete (closed) rigid annuloplasty rings are superior to partial (i.e., open) flexible band because rigid rings have less $M R$ recurrence over time. Spoor et al. retrospectively reviewed outcomes in 289 patients with LVEF $\leq 30 \%$ who received an undersized complete mitral annuloplasty ring for MV repair in the setting of ischemic or idiopathic cardiomyopathy. 170 patients had a flexible complete ring and 119 patients received a 26 or $28 \mathrm{~mm}$ undersized nonflexible complete ring. The need for repeat procedures due to significant recurrent MR was $9.4 \%$ in the flexible group (average time to reoperation $2.4 \mathrm{yrs}$ ), vs. $2.5 \%$ in the nonflexible group (average time to reoperation $4.0 \mathrm{yrs}$ ) (6).

The mechanism of action of the IRIS device is unique and there is no other transcatheter device currently available with the same function. The device is a semi-rigid complete ring which attaches directly to the entire mitral annulus and can normalize the mitral anterior-posterior diameter in properly selected patients. The device is supra-annular and there is no interference with the left ventricular outflow tract. It has previously been shown that normalization of the SL diameter is one of the most important effects in restoring leaflet coaptation and abolishing ischemic MR (7). The Millipede IRIS system has been effective at normalizing the SL diameter of the mitral annulus in the clinical experience to date.

As previously reported, there was a reduction in average mitral SL diameter from 38.0 to $25.9 \mathrm{~mm}$, representing an average SL reduction of $31.8 \%$ (3). The Millipede IRIS ring can also be used in conjunction with transcatheter edge-to-edge repair with the MitraClip device (8). It is also intriguing to consider that the IRIS ring could serve as a "docking station" for subsequent transcatheter mitral valvein-ring implantation.

\section{Summary}

The Millipede IRIS mitral annuloplasty ring is the first transcatheter complete semi-rigid ring to be delivered through a transfemoral, transseptal approach to the mitral annulus. Further device, delivery system and intraprocedural imaging improvements are ongoing and the device will be further evaluated in ongoing clinical trials. 

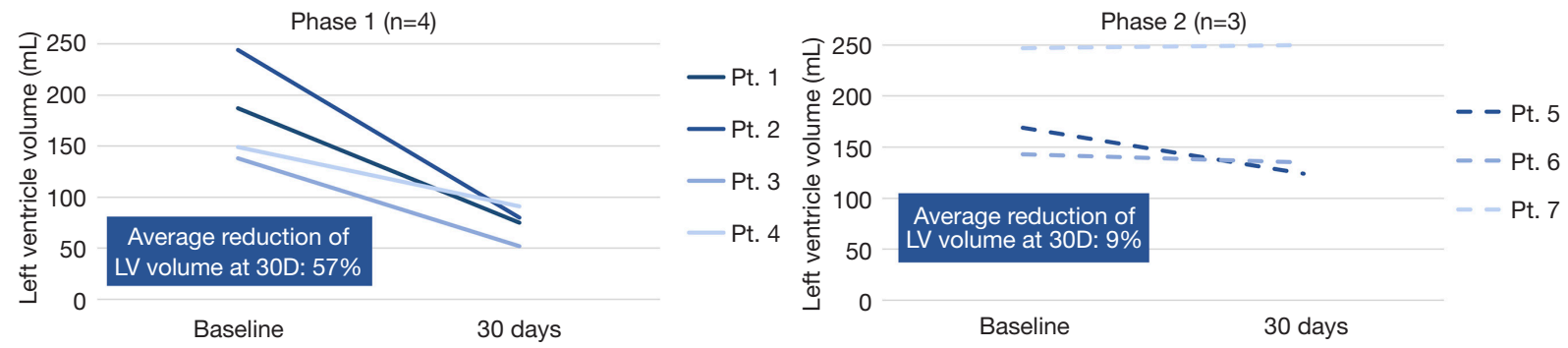

Figure 8 Reduction in left ventricular volume. Phase 1, surgical implants; Phase 2, transcatheter implants. Reproduced with permission (3).

\section{Acknowledgements}

None.

\section{Footnote}

Conflicts of Interest: Drs. Bolling, Boyd, Rogers and Smith are consultants to Millipede, Inc.

\section{References}

1. Carpentier A. Reconstructive valvuloplasty. A new technique of mitral valvuloplasty. Presse Med 1969;77:251-3.

2. Iung B, Baron $\mathrm{G}$, Butchart EG, et al. A prospective survey of patients with valvular heart disease in Europe: The Euro Heart Survey on Valvular Heart Disease. Eur Heart J 2003;24:1231-43.

3. Rogers JH, Boyd WD, Smith TW, et al. Transcatheter Annuloplasty for Mitral Regurgitation with an Adjustable Semi-Rigid Complete Ring: Initial Experience with the

Cite this article as: Rogers JH, Boyd WD, Smith TW, Bolling SF. Early experience with Millipede IRIS transcatheter mitral annuloplasty. Ann Cardiothorac Surg 2018;7(6):780-786. doi: 10.21037/acs.2018.10.05
Millipede IRIS Device. Structural Heart 2018;2:43-50.

4. Stone GW, Vahanian AS, Adams DH, et al. Clinical

Trial Design Principles and Endpoint Definitions for Transcatheter Mitral Valve Repair and Replacement: Part 1: Clinical Trial Design Principles: A Consensus Document From the Mitral Valve Academic Research Consortium. J Am Coll Cardiol 2015;66:278-307.

5. Gillinov AM, Cosgrove DM, Blackstone EH, et al. Durability of mitral valve repair for degenerative disease. J Thorac Cardiovasc Surg 1998;116:734-43.

6. Spoor MT, Geltz A, Bolling SF. Flexible versus nonflexible mitral valve rings for congestive heart failure: differential durability of repair. Circulation 2006;114:I67-71.

7. Timek TA, Lai DT, Tibayan F, et al. Septal-lateral annular cinching abolishes acute ischemic mitral regurgitation. J Thorac Cardiovasc Surg 2002;123:881-8.

8. Rogers JH, Boyd WD, Smith TWR, et al. Combined MitraClip Edge-to-Edge Repair With Millipede IRIS Mitral Annuloplasty. JACC Cardiovasc Interv 2018;11:323-4. 